\title{
Solid angle subtended by a cylindrical detector at a point source in terms of elliptic integrals
}

\author{
M. J. Prata ${ }^{1}$ \\ Instituto Tecnológico e Nuclear (ITN), Sacavém, Portugal
}

\begin{abstract}
The solid angle subtended by a right circular cylinder at a point source located at an arbitrary position generally consists of a sum of two terms: that defined by the cylindrical surface $\left(\Omega_{c y l}\right)$ and the other by either of the end circles $\left(\Omega_{c i r c}\right)$. We derive an expression for $\Omega_{c y l}$ in terms of elliptic integrals of the first and third kinds and give similar expressions for $\Omega_{\text {circ }}$ using integrals of the first and second kinds. These latter can be used alternatively to an expression also in terms of elliptic integrals, due to Philip A. Macklin and included as a footnote in Masket (Rev. Sci. Instr., 28 (3), 191-197, 1957). The solid angle subtended by the whole cylinder when the source is located at an arbitrary location can then be calculated using elliptic integrals.
\end{abstract}

Key words: solid angle, point source, cylindrical detector, cylinder, elliptic integrals

\section{Introduction}

The knowledge of the solid angle $(\Omega)$ subtended by a right, finite, circular cylinder at a point isotropic source is required in numerous problems in nuclear and radiation physics. Generally, $\Omega$ can be expressed as sum of two components: that subtended by the cylindrical surface $\left(\Omega_{c y l}\right)$ and the other by either of the end circles $\left(\Omega_{\text {circ }}\right)$. Through the years this calculation has been addressed by various authors using different methods. Without the worry of

Email address: mjprata@sapo.pt (M. J. Prata).

1 Partially supported by Fundação para a Ciência e Tecnologia (Programa Praxis XXI - BD/15808/98)

Preprint submitted to Elsevier Science 31 May 2018 
being exhaustive we give some examples of such works. Masket (1957) outlined a general procedure based on Stokes theorem to reduce the double integral $\Omega=\iint \sin \theta d \theta d \varphi$ to a contour integral in a single variable $(\theta$ or $\varphi)$. The method was used to express $\Omega_{c i r c}$ and $\Omega_{c y l}$ as single integrals which were numerically integrated. Extensive tables describing these results both for the disc and the entire cylinder were reported in a separate work (Masket et al, 1956). An approximation to the solid angle defined by two parallel plane surfaces was described by Gillespie (1970) and applied in the cases of two equal rectangles and two equal circles in a face-to-face geometry. With this method each surface is subdivided into small finite areas and the two double integrals are then replaced by a double summation. The calculation of $\Omega_{\text {circ }}$ was treated by Gardner and Verghese (1971) by replacing the disc with a regular n-side polygon of equal area, for which an analytical expression was given. In a similar way, $\Omega_{c y l}$ was approximated by the analytical expression for an n-side regular polyhedral surface (Verghese et al, 1972). Green et al (1974) used the Monte Carlo method to calculate the cylinder solid angle for two height-toradius ratios $(1: 1,2: 1)$, considering distances from source to cylinder center up to 12 cylinder radii and angular positions of the source ranging from $0^{\circ}$ to $90^{\circ}$ from the cylinder axis.

An analytical expression for $\Omega_{\text {circ }}$ in terms of elliptic integrals due to Philip A. Macklin (Macklin, 1957) appears included as a footnote in Masket (1957). In the present work we show that also $\Omega_{c y l}$ can be reduced to elliptic integrals and give, without derivation, expressions for $\Omega_{c i r c}$ which can be deduced in a akin way and are different from that due to P.A. Macklin. The solid angle of the whole cylinder can then be expressed in terms of elliptic integrals which are rather well known functions (e.g. Milne-Thomson, 1964) for which computation algorithms and tables are readily available.

Instead of blind numerical integration, one can turn to the variety of numeric methods already existent, which enable the fast calculation of the solid angle for the whole range of parameters. For instance, the complete integrals of the first and second kinds can be computed using (i) the polynomial approximations due to Hastings (1955) and included in Milne-Thomson (1964, eqs. 17.3.33 to 17.3.36); (ii) the process of the arithmetic-geometric mean (Milne-Thomson, 1964, 17.6) or (iii) the infinite series (Milne-Thomson, 1964, 17.3.11, 17.3.12) which can be used in combination with Landen's transformation when the modular angle is close to $\pi / 2$.

Since the solid angle can be decomposed into elliptic integrals, any possibility of finding general analytical expressions in terms of elementary functions is henceforward precluded. On the other hand, the calculation has been put under the sound roof of the subject of elliptic integrals and functions.

In a recent work (Prata, 2002) we describe the analytical calculation of the 
solid angle subtended by a cylinder at a point cosine source. Combining these results with those presented here, the case of an axially symmetric point source with an angular distribution given by $f_{k}(\boldsymbol{\Omega})=1+a \boldsymbol{\Omega} \cdot \mathbf{k}$ can be treated analitically when the source axis $(\mathbf{k})$ is orthogonal to that of the cylinder.

\section{Solid Angle Calculation}

The solid angle subtended by a given surface at a point isotropic source can be defined as

$$
\Omega_{\text {surf }}=\frac{1}{4 \pi} \underset{\begin{array}{c}
\text { directions } \\
\text { hitting surface }
\end{array}}{\iint_{\text {suce }}} d \Omega,
$$

so that $0 \leq \Omega_{\text {surf }} \leq 1$. As previously said, in the case of a circular cylinder, the solid angle $\Omega$ is in general given by $\Omega=\Omega_{c y l}+\Omega_{\text {circ }}$, where $\Omega_{c y l}$ and $\Omega_{\text {circ }}$ are the contributions of the cylindrical surface and of one of the end circles. These quantities can be defined with the help of figs. 1 and 2, where the source is located at the origin of the coordinate system and the $z$ axis is both parallel to the cylinder axis and orthogonal to the planes of the circles. To calculate $\Omega_{c y l}$ it is sufficient to consider the situation depicted in fig. 1, where the source lies

in one of the planes delimiting the cylindrical surface. Let $\Omega_{c y l 0}(L, r, d)$ denote the solid angle in this case and $\Omega_{\text {circ }}(L, r, d)$ the solid angle defined by the disc. Let us illustrate how $\Omega$ can be calculated in an arbitrary situation. Referring again to fig. 1 , but assuming that the source is located at $(0,0,-|z|)$, one has $\Omega=\Omega_{c y l 0}(L+|z|, r, d)-\Omega_{c y l 0}(|z|, r, d)+\Omega_{c i r c}(|z|, r, d)$. If the source is located at $(0,0, z)$ and $0<z<L$, then $\Omega=\Omega_{c y l 0}(z, r, d)+\Omega_{c y l 0}(L-z, r, d)$. If $r>d$ and $z<0$ or $z>L$, the solid angle is defined by one of the circles alone, e.g. $\Omega=\Omega_{\text {circ }}(|z|, r, d)$ for $z<0$.

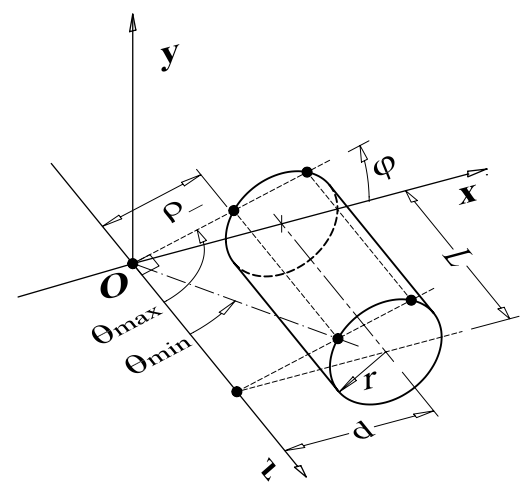

Fig. 1. Notation for $\Omega_{c y l 0}$ 


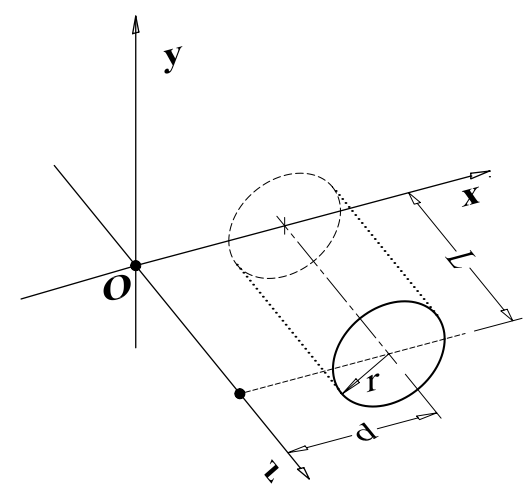

Fig. 2. Notation for $\Omega_{\text {circ }}$

\subsection{Calculation of $\Omega_{\text {cyl0 }}$}

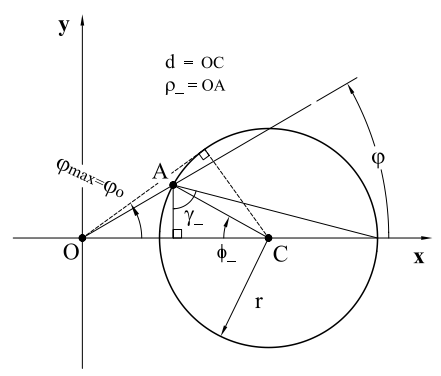

Fig. 3. Quantities used to calculate $\Omega_{c y l 0}$

From figs. 1 and 3, it follows that

$\Omega_{c y l 0}(L, r, d)=1 /(4 \pi) \int_{\varphi_{\min }}^{\varphi_{\max }} \int_{\theta_{\min }} \sin \theta d \theta d \varphi=1 /(2 \pi) \int_{0}^{\varphi_{o}}\left(\cos \theta_{\min }-\cos \theta_{\max }\right) d \varphi$

where

$$
\varphi_{\max }=-\varphi_{\min }=\varphi_{o} \equiv \arcsin (r / d)
$$

$\cos \theta_{\min }=L / \sqrt{L^{2}+\rho_{-}^{2}(\varphi)}$

$\cos \theta_{\max }=0$

and

$$
\rho_{-}(\varphi)=d \cos \varphi-\sqrt{r^{2}-(d \sin \varphi)^{2}}
$$


Thus,

$$
\Omega_{c y l 0}=L /(2 \pi) \int_{0}^{\varphi_{o}}\left[L^{2}+\rho_{-}^{2}(\varphi)\right]^{-1 / 2} d \varphi
$$

Then we change the integration variable to $\gamma_{-}$represented in fig. 3 and given by

$$
\gamma_{-}=\pi / 2-\phi_{-} / 2
$$

where

$\phi_{-} / 2=\arctan \left[\sin (\varphi) \rho_{-} /\left(r+d-\cos (\varphi) \rho_{-}\right)\right]$

and $\rho_{-}(\varphi)$ is obtained from eq. 3 .

Eq. 4 is rewritten as

$$
\Omega_{c y l 0}=L /(2 \pi) \int_{\gamma_{o}}^{\pi / 2} \frac{1}{\sqrt{L^{2}+\rho_{-}^{2}\left(\gamma_{-}\right)}}\left[\frac{d^{2}-r^{2}}{\rho_{-}^{2}\left(\gamma_{-}\right)}-1\right] d \gamma_{-}
$$

where

$$
\rho_{-}\left(\gamma_{-}\right)=\sqrt{(d+r)^{2}-4 d r \sin ^{2} \gamma_{-}}
$$

and

$$
\gamma_{o}=\left(\pi / 2+\varphi_{o}\right) / 2
$$

Introducing

$$
\begin{gathered}
m=4 r d /\left(L^{2}+(d+r)^{2}\right), \\
n=4 r d /(d+r)^{2},
\end{gathered}
$$

there results from eq. 7 :

$\rho_{-}^{2}\left(\gamma_{-}\right)=(d+r)^{2}\left(1-n \sin ^{2} \gamma_{-}\right)$ 
$\sqrt{L^{2}+\rho_{-}^{2}\left(\gamma_{-}\right)}=\sqrt{L^{2}+(d+r)^{2}} \sqrt{1-m \sin ^{2} \gamma_{-}}$,

$L / \sqrt{L^{2}+(d+r)^{2}}=\sqrt{1-m / n}$

and

$(d-r) /(d+r)=\sqrt{1-n} ; d \geq r$.

Substituting in the rhs of eq. 6 yields

$\Omega_{c y l 0}=1 /(2 \pi) \sqrt{1-m / n} \int_{\gamma_{o}}^{\pi / 2} \frac{1}{\sqrt{1-m \sin ^{2} \gamma_{-}}}\left[\frac{\sqrt{1-n}}{\left(1-n \sin ^{2} \gamma_{-}\right)}-1\right] d \gamma_{-}$.

The integral is easily decomposed into elliptic integrals in the Legendre form:

$\Omega_{c y l 0}=1 /(2 \pi) \sqrt{1-m / n}\left\{\sqrt{1-n}\left[\pi(n ; m)-\pi\left(n ; \gamma_{o} \mid m\right)\right]-\left[K(m)-F\left(\gamma_{o} \mid m\right)\right]\right\}$

where $F\left(\gamma_{o} \mid m\right), \pi\left(n ; \gamma_{o} \mid m\right)$ are the incomplete elliptic integrals of the first and third kinds, respectively; and $K(m)=F(\pi / 2 \mid m), \pi(n ; m)=\pi(n ; \pi / 2 \mid m)$ are the corresponding complete integrals (Milne-Thomson, 1964). From eqs. 9 and 10 it is clear that the parameter $m$ and the characteristic $n$ verify

$$
\begin{gathered}
0 \leq m \leq 1,(m=1 \Leftrightarrow L=0 \wedge d=r), \\
0 \leq n \leq 1,(n=1 \Leftrightarrow d=r)
\end{gathered}
$$

and

$$
0 \leq m \leq n \leq 1 ;(m=n \Leftrightarrow L=0)
$$

For computational purposes it is worth mentioning that, because of eq. 14, both $\pi\left(n ; \gamma_{o} \mid m\right)$ and $\pi(n ; m)$ in the rhs of eq. 11 fall into the the circular case (Milne-Thomson, 1964, 17.7.11), except when $L=0$ or $d=r$. These special cases are treated separately in section 2.3 .

\subsection{1 $\Omega_{c y l 0}$ when $d \gtrsim r$}

From eqs. 8 and 2 results that, as $d \rightarrow r^{+}, \gamma_{o} \rightarrow \pi / 2$ and the two subtractions

of elliptic integrals in the rhs of eq. 11 approach zero (e.g. $K(m)-F\left(\gamma_{o} \mid m\right) \rightarrow$ 
0 ). To avoid the consequent loss of precision, an approximation ${ }^{2}$ (Masket, 1957, eq. 32) can be used:

$$
\begin{aligned}
\Omega_{c y l 0} & =(2 \pi)^{-1}\left\{\varphi_{o}-1 / 2\left[r d \cos \varphi_{o}-r^{2}\left(\pi / 2-\varphi_{o}\right)\right] L^{-2}\right. \\
& \left.+3 / 8\left[r d\left(d^{2}+2 r^{2}\right) \cos \varphi_{o}-r^{2}\left(r^{2}+2 d^{2}\right)\left(\pi / 2-\varphi_{o}\right)\right] L^{-4}-\ldots\right\}
\end{aligned}
$$

where the cancellations can be more easily dealt with. Eq. 15 results from expanding and termwise integrating the integrand on the rhs of eq. 4. Since $d-r \leq \rho_{-}(\varphi) \leq \sqrt{d^{2}-r^{2}}$ when $0 \leq \varphi \leq \varphi_{o}$, the expansion can safely be done for $\sqrt{d^{2}-r^{2}}<L$ and eq. 15 gives a good approximation (better than four digits) for $\sqrt{d^{2}-r^{2}}<L / 10$.

\subsection{Expressions for $\Omega_{\text {circ }}$}

In a similar fashion to that used to obtain $\Omega_{c y l 0}$ one can show that the solid angle subtended by a circular disc (fig. 2) can be written using complete elliptic integrals of the third and first kinds:

$$
\begin{aligned}
& \Omega_{c i r c}(L, r, d)=(2 \pi)^{-1} \sqrt{1-m / n}\{\sqrt{1-n} \pi(n ; \pi / 2 \mid m)-K(m)\} ; d>r \quad(16) \\
& \Omega_{c i r c}(L, r, d)=1 / 2-(2 \pi)^{-1} \sqrt{1-m / n}\{\sqrt{1-n} \pi(n ; \pi / 2 \mid m)+K(m)\} ; d<r
\end{aligned}
$$

where $m, n$ are obtained from eqs. 9, 10 .

The complete integral of the third kind can be expressed in terms of incomplete integrals of the first and second kinds which are easier to calculate. Since $0 \leq m \leq n \leq 1$ one can use (Milne-Thomson, 1964, 17.7.14,17.4.40) to show that:

$$
\begin{gathered}
\Omega_{c i r c}=1 / 4-(2 \pi)^{-1} n /(1+\sqrt{1-n}) \sqrt{1-m / n} K(m) \\
-(2 \pi)^{-1}\left\{[E(m)-K(m)] F\left(\epsilon \mid m^{\prime}\right)+K(m) E\left(\epsilon \mid m^{\prime}\right)\right\}, d>r,
\end{gathered}
$$

$\overline{2}$ We include here a normalizing factor of $1 /(4 \pi)$. 


$$
\begin{gathered}
\Omega_{c i r c}=1 / 4-(2 \pi)^{-1}(1+\sqrt{1-n}) \sqrt{1-m / n} K(m) \\
+(2 \pi)^{-1}\left\{[E(m)-K(m)] F\left(\epsilon \mid m^{\prime}\right)+K(m) E\left(\epsilon \mid m^{\prime}\right)\right\}, d<r,
\end{gathered}
$$

where

$$
\begin{gathered}
m^{\prime}=1-m, \\
\epsilon=\arcsin \sqrt{(1-n) /(1-m)},
\end{gathered}
$$

$E(\varphi \mid m)$ is the elliptic integral of the second kind and $E(m)=E(\pi / 2 \mid m)$ is the complete integral.

As an alternative to eqs. 18, 19, one can use an expression ${ }^{3}$ due to Macklin (1957) which appears as a footnote in Masket (1957):

$$
\begin{gathered}
(4 \pi) \Omega_{\text {circ }}(L, r, d)=2 \pi+2[K(m)-E(m)]\left[F\left(\theta \mid m^{\prime}\right)+F\left(\psi \mid m^{\prime}\right)\right] \\
-2 K(m)\left\{E\left(\theta \mid m^{\prime}\right)+E\left(\psi \mid m^{\prime}\right)+2 \beta /\left(\left[1+(\alpha+\beta)^{2}\right]^{\frac{1}{2}}\left[\beta+\left(1+\alpha^{2}\right)^{\frac{1}{2}}\right]\right)\right\},
\end{gathered}
$$

where

$\theta=\arcsin \frac{\left[1+(\alpha+\beta)^{2}\right]^{\frac{1}{2}}}{\beta+\left(1+\alpha^{2}\right)^{\frac{1}{2}}}, \quad \psi=\arcsin \frac{\left(1+\alpha^{2}\right)^{\frac{1}{2}}-\beta}{\left[1+(\alpha-\beta)^{2}\right]^{\frac{1}{2}}}$,

$0 \leq \theta \leq \pi / 2, \quad-\pi / 2 \leq \psi \leq \pi / 2, \quad \theta \geq|\psi|$

$0 \leq \alpha=d / L \leq \infty, \quad 0 \leq \beta=r / L \leq \infty$

and $m, m \prime$ are obtained from eqs. 9 and 20.

\subsection{Special values and continuity}

Since $K(m=1)=\infty$ and $\pi(n ; \pi / 2 \mid m)=\infty$ whenever $n=1$ or $m=1$, the cases $L=0$ and $d=r$ have to be studied separately (see eqs. 12 and 13).

3 We introduce a factor of $4 \pi$ to conform to the normalization used in the present work. 
Starting from eq. 4 it is straightforward that $\Omega_{c y l 0}\left(d \longrightarrow r^{+}, L \neq 0\right)=1 / 4$. Since $\Omega_{c y l 0}(d>r, L=0)=0, \Omega_{c y l 0}$ is discontinuous when $d \longrightarrow r^{+}, L \longrightarrow 0$. Using the properties of elliptic integrals (Milne-Thomson, 1964) one can easily show from eqs. 18 and 19 that:

$$
\begin{gathered}
\Omega_{\text {circ }}\left(d \longrightarrow r^{ \pm}, L \neq 0\right)=1 / 4-(2 \pi)^{-1} \sqrt{1-m_{1}} K\left(m_{1}\right), \\
\Omega_{c i r c}(L \longrightarrow 0)=\{0(d>r) ; 1 / 4(d=r) ; 1 / 2(d<r)\}
\end{gathered}
$$

where $m_{1}=\left.m\right|_{r=d}=4 r^{2} /\left(L^{2}+4 r^{2}\right)$. $\Omega_{\text {circ }}$ is thus continuous except for $L=0$. Eq. 23 is the same as Masket (1957, eq. 24), divided by a normalizing factor of $4 \pi$.

\section{Summary and outlook}

An expression for $\Omega_{c y l}$ in terms of complete and incomplete elliptic integrals of the first and third kinds has been derived in the case where the source lies in the same plane as one of the end discs (eq. 11 ). Expressions for $\Omega_{\text {circ }}$ decomposed into complete and incomplete elliptic integrals of the first and second kinds using a single amplitude $(\epsilon)$ were given (eqs. 18 and 19). These expressions can be used alternatively to one (eq.22) due to Macklin (1957) containing also complete and incomplete elliptic integrals of the first and second kinds but using two amplitudes $(\theta, \psi)$. Computational methods for the elliptic integrals can be found in Milne-Thomson (1964). The required third kind integrals all belong to the circular case $(0 \leq m<n<1)$ except for a few cases which are treated in section 2.3. The solid angle when the source is at an arbitrary position can be calculated using the expressions referred, as described in the beginning of section 2 .

The solid angle defined by a right circular cylinder and an isotropic source distributed on a wire parallel to the cylinder axis can also be decomposed into elliptic integrals in a way akin to that used here to obtain $\Omega_{c y l 0}$. A work where we report this result is in preparation.

\section{Acknowledgements}

Thanks are due to João Prata for reviewing this manuscript. I would like to thank Professor John H. Hubbell for providing a copy of the work by A.V. Masket (Masket, 1957). 


\section{References}

Gardner, R.P. \& Verghese, K., 1971. On the solid angle subtended by a circular disc. Nucl. Instr. Meth. 93, 163-167.

Gillespie, C.R., 1970. Determination of the geometrical factor of cylindrical geometries. Rev. Sci. Instr. 41 (1), 42-43.

Green, M.V., Aamodt, R.L. \& Johnston, G.S., 1974. The solid angle subtended by a solid, right, circular cylinder as seen from a point in space. Nucl. Instr. Meth. 117, 409-412.

Hastings, C., 1955. Approximations for digital computers, Princeton Univ. Press, Princeton, N.J.

Macklin, P.A., 1957. Expression for the solid angle subtended by a circular disc at a point source in terms of elliptic integrals. Included as a footnote in Masket (1957).

Masket, A.V., Macklin, R.L. \& Schmitt, H.W., 1956. Tables of solid angle values and activations. ORNL-2170 (Oak Ridge Nat. Lab., Oak Ridge, Tenn.)

Masket, A.V., 1957. Solid Angle contour integrals, series, and tables. Rev. Sci. Instr. 28 (3), 191-197.

Milne-Thomson, L.M., 1964. Elliptic integrals. In: Abramowitz, M., Stegun, I. A. (Eds.), Handbook of mathematical functions (9th Printing), Dover Publications Inc., N. York, pp. 589-627.

Prata, M.J., 2002. Analytical calculation of the solid angle defined by a cylindrical detector and a point cosine source with orthogonal axes. Accepted for publication in Rad. Phys. Chem.

Verghese, K., Gardner, R.P., \& Felder, R.M., 1972. Solid angle subtended by a circular cylinder. Nucl. Instr. Meth. 101, 391-393. 